Communications in Physics, Vol. 22, No. 3 (2012), pp. 223-228

\title{
MOTT TRANSITIONS IN THE 2-BAND HUBBARD MODEL: A COHERENT POTENTIAL APPROXIMATION STUDY
}

\author{
LE DUC ANH \\ Department of Physics, Hanoi National University of Education \\ HOANG ANH TUAN \\ Institute of Physics, VAST
}

\begin{abstract}
The half-filled isotropic degenerate two-band Hubbard model is studied within coherent potential approximation. The model is characterized by an Ising-type Hunds exchange coupling, intra- and inter-orbital Coulomb parameters. We found that the band degeneracy slightly reduces the Mott-Hubbard critical value $U_{C}$. We reveal that the system can have two distinct critical values for Mott-Hubbard transitions.
\end{abstract}

\section{INTRODUCTION}

The one-band Hubbard model has been used as a model for a study of strongly correlated electronic systems such as transition metal, valence-mixing and hight $T_{C}$ materials. Several quantitative comparisons between the physics of three dimensional transition metal oxides and the one-band Hubbard model give surprisingly good agreement $[1,2]$. This good agreement is questionable since, normally, the simplest description of a real system involves electrons in a two(or more)-fold degenerate level. Using slave boson (SB) technique, Hasegawa [3] showed that the band degeneracy has strong effect on the MottHubbard transitions. The critical value $U_{C}$ of the double degenerate case is 1.33 times larger than that of the one-band model. Furthermore, Ono, Potthoff, and Bulla with an extension of the linearized dynamical mean-field theory found a linear dependence of $U_{C}$ on the band degeneracy [4]. In contrast, using dynamical mean-field with iterative perturbation theory Kajueter and Kotliar [5] pointed out that the critical value $U_{C}$ was slightly reduced by the band degeneracy. The purpose of this report is to study Mott-Hubbard transitions in the doubly degenerate Hubbard model at half-filling using the coherent potential approximation (CPA) [6-8]. This self-consistent approximation is known to be very successful in explaining single-particle properties of disordered systems and is well suited to study the usual Hubbard model [9]. In studying the Hubbard model the CPA has advantages over the SB and DMFT of being analytically simple. It provides some analytical results and does not require much computer work. 


\section{MODEL AND FORMALISM}

The two-band Hubbard model reads

$$
\begin{aligned}
H= & t \sum_{<i j>\alpha \sigma}\left[f_{i \alpha \sigma}^{+} f_{j \alpha \sigma}+\text { h.c. }\right]+U \sum_{i \alpha} n_{i \alpha \uparrow} n_{i \alpha \downarrow} \\
& +U^{\prime} \sum_{i \alpha \bar{\alpha}} n_{i \alpha \uparrow} n_{i \bar{\alpha} \downarrow}+\left(U^{\prime}-J\right) \sum_{i \sigma} n_{i 1 \sigma} n_{i 2 \sigma} .
\end{aligned}
$$

Here $f_{i \alpha \sigma}^{+}\left(f_{i \alpha \sigma}\right)$ is creation (annihilation) operator for an electron at site i with spin $\sigma$ in the band $\alpha ; t$ is hopping parameter between nearest-neighbor sites; intra- and interorbital Coulomb repulsion parametrized by $U$ and $U^{\prime}$, respectively; $J$ is Ising-type Hunds exchange coupling; $n_{i \alpha \sigma}=f_{i \alpha \sigma}^{+} f_{i \alpha \sigma}$ for spin $\sigma \in\{\uparrow, \downarrow\}$.

We apply coherent potential approximation (CPA) to the above model. One starts from an intuitive physical picture: an electron with spin $\sigma$ can hop onto the $\alpha$-orbital situated at site $i$, if the orbital is either empty or occupied by an electron with spin $\bar{\sigma}$. In addition, due to the exchange and interorbital interations, the energy level of the electron also depends on number of electrons occupied on the $\bar{\alpha}$ orbital, i.e., orbital configurations. Thus, the electron is considered as moving in a static random potential with eigenvalues $\varepsilon$ and probabilities $P$ given by Table I [8]. The effective Hamiltonian is

$$
H=t \sum_{<i j>\alpha \sigma}\left[f_{i \alpha \sigma}^{+} f_{j \alpha \sigma}+h . c .\right]+\sum_{i \alpha \sigma} \sum_{\alpha \sigma}(\omega) f_{i \alpha \sigma}^{+} f_{i \alpha \sigma},
$$

where $\sum_{\alpha \sigma}(\omega)$ is CPA self-energy for spin $\sigma$ at orbital $\alpha$ that is determined by demanding the scattering matrix for a carrier at an arbitrarily chosen site embedded in the effective medium vanished on average. One thus obtains the average Green function

$$
G_{\alpha \sigma}(z)=\sum_{\lambda=1}^{8} P_{\alpha \sigma}^{\lambda} G_{\alpha \sigma}^{\lambda}(z) .
$$

Here the configurational probabilities $P_{\alpha \sigma}^{\lambda}$ are calculated via the partial Green functions $G_{\alpha \sigma}^{\lambda}(z)$ (see Ref. [10])).

$$
G_{\alpha \sigma}^{\lambda}(z)=\frac{G_{\alpha \sigma}(z)}{1+\left(\sum_{\alpha \sigma}(z)-\varepsilon_{\lambda}\right) G_{\alpha \sigma}(z)}
$$

Finally, the system of equations is close by requiring that the Green function of the effective medium coincides within the lattice Green function:

$$
G_{\alpha \sigma}(z)=\int \frac{\rho_{0}(\omega)}{z-\sum_{\alpha \sigma}(z)-\omega} d \omega
$$

where $\rho_{0}(\omega)$ is the bare density of states (DOS).

\section{RESULTS AND DISCUTIONS}

The self-energy and the Green function are obtained by iterations [7] which can be done for arbitrary lattice structure. Here for convenient we chose $\rho_{0}(\omega)$ is the DOS of the 
Table 1. Energy levels $\epsilon_{1 \uparrow}^{\lambda}$ and configuration probabilities $P_{1 \uparrow}^{\lambda}$ for a $1 \uparrow$ electron. $U, U^{\prime}$, and $J$ are the intraorbital Coulomb interaction, the interorbital Coulomb interaction, and the exchange interaction, respectively. $n_{\alpha \sigma}$ is the average occupation of electrons with spin $\sigma$ on the $\alpha$ orbital. Similar tables exist for the $1 \downarrow$, $2 \downarrow$, and $2 \uparrow$ electrons. Taken from Ref. [8].

\begin{tabular}{|c|c|c|c|c|c|l|}
\hline Config. & \multicolumn{3}{|c|}{ Orbitals occupation } & Energy & Probabilities \\
$\lambda$ & $1 \uparrow$ & $1 \downarrow$ & $2 \uparrow$ & $2 \downarrow$ & $\varepsilon_{1 \uparrow}^{\lambda}$ & $P_{1 \uparrow}^{\lambda}$ \\
\hline 1 & 1 & 0 & 0 & 0 & 0 & $\left\langle\left(1-n_{1 \downarrow}\right)\left(1-n_{2 \uparrow}\right)\left(1-n_{2 \downarrow}\right)\right\rangle$ \\
\hline 2 & 1 & 0 & 1 & 0 & $U^{\prime}-J$ & $\left\langle\left(1-n_{1 \downarrow}\right) n_{2 \uparrow}\left(1-n_{2 \downarrow}\right)\right\rangle$ \\
\hline 3 & 1 & 0 & 0 & 1 & $U^{\prime}$ & $\left\langle\left(1-n_{1 \downarrow}\right)\left(1-n_{2 \uparrow}\right) n_{2 \downarrow}\right\rangle$ \\
\hline 4 & 1 & 1 & 0 & 0 & $U$ & $\left\langle n_{1 \downarrow}\left(1-n_{2 \uparrow}\right)\left(1-n_{2 \downarrow}\right)\right\rangle$ \\
\hline 5 & 1 & 0 & 1 & 1 & $2 U^{\prime}-J$ & $\left\langle\left(1-n_{1 \downarrow}\right) n_{2 \uparrow} n_{2 \downarrow}\right\rangle$ \\
\hline 6 & 1 & 1 & 1 & 0 & $U+U^{\prime}-J$ & $\left\langle n_{1 \downarrow} n_{2 \uparrow}\left(1-n_{2 \downarrow}\right)\right\rangle$ \\
\hline 7 & 1 & 1 & 0 & 1 & $U+U^{\prime}$ & $\left\langle n_{1 \downarrow}\left(1-n_{2 \uparrow}\right) n_{2 \downarrow}\right\rangle$ \\
\hline 8 & 1 & 1 & 1 & 1 & $U+2 U^{\prime}-J$ & $\left\langle n_{1 \downarrow} n_{2 \uparrow} n_{2 \downarrow}\right\rangle$ \\
\hline
\end{tabular}

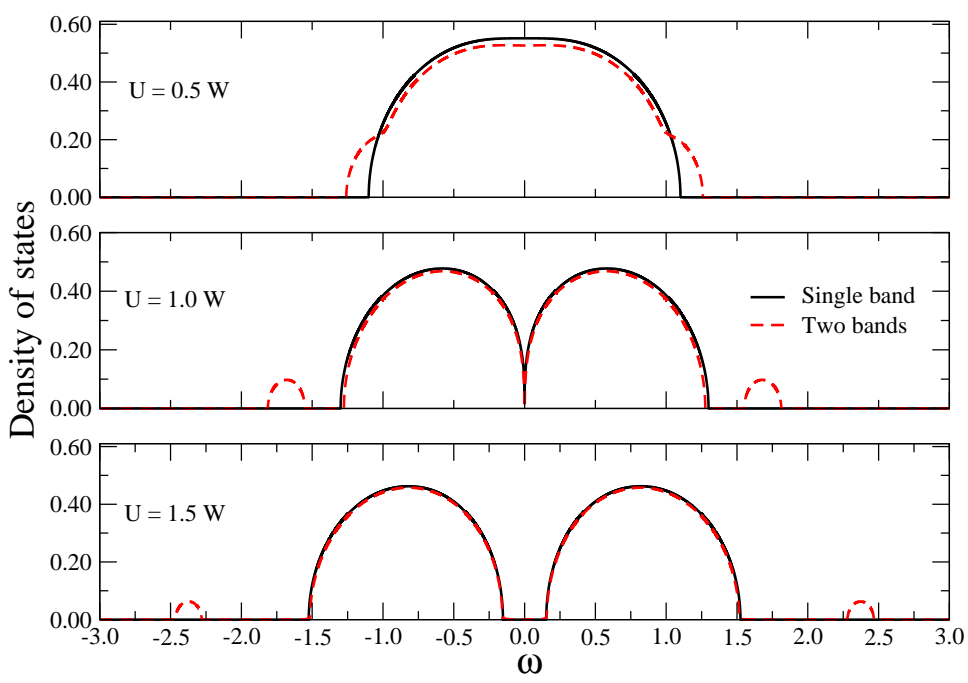

Fig. 1. DOS of the one-band $\left(U^{\prime}=0\right)$ and two-band $\left(U^{\prime}=U\right)$ models for $\mathrm{J}=0$ and different values of $U$.

Bethe lattice at infinite dimension:

$$
\rho_{0}(\varepsilon)=\frac{2}{\pi W^{2}} \sqrt{W^{2}-\varepsilon^{2}} .
$$

Since the system is isotropic the Fermi energy $E_{F}$ is exactly located at the origin. The Mott transitions appear simutanously on two orbitals. In order to show the effect of the band degeneracy, we compare results of the single-band model $\left(U^{\prime}=0, J=0\right)$ with that of the two-band models $\left(U^{\prime}=U, J=0\right)$. Fig. 1 shows the DOS of the single band and two-band models for various values of $U$. It is seen that the band degeneracy 


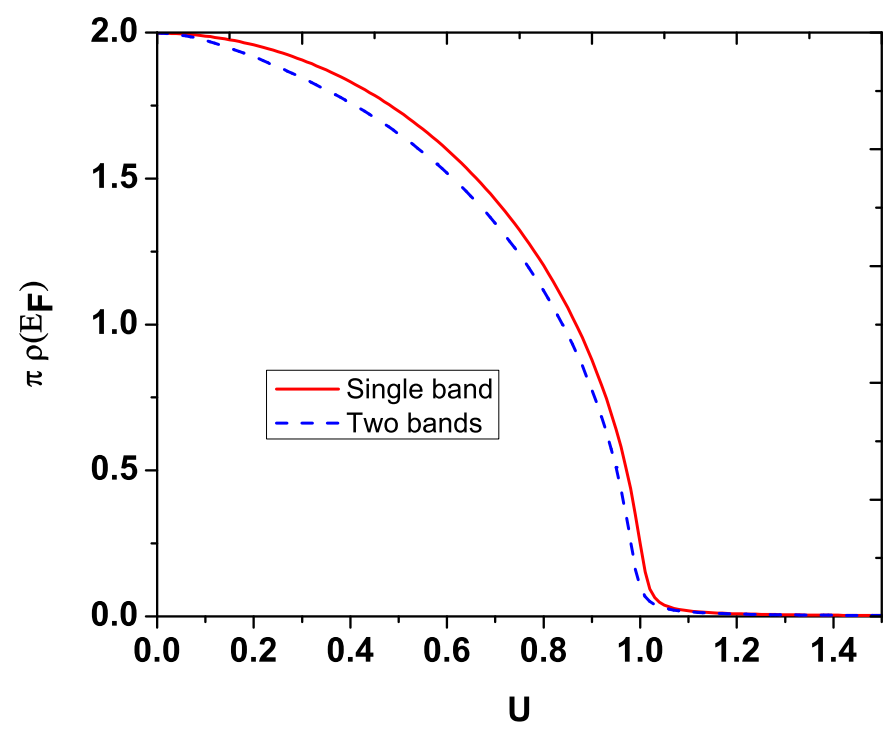

Fig. 2. Mott-Hubbard transitions in the one- and two-band models.

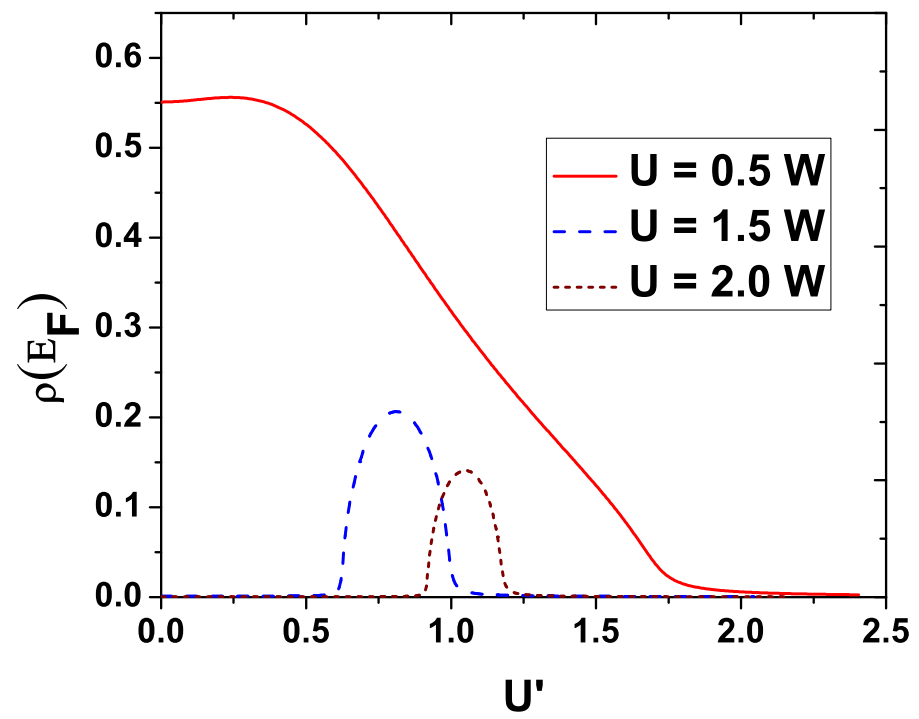

Fig. 3. DOS at the Fermi level as a function of $U^{\prime}$ for $J=0$ and different values of $U$. 


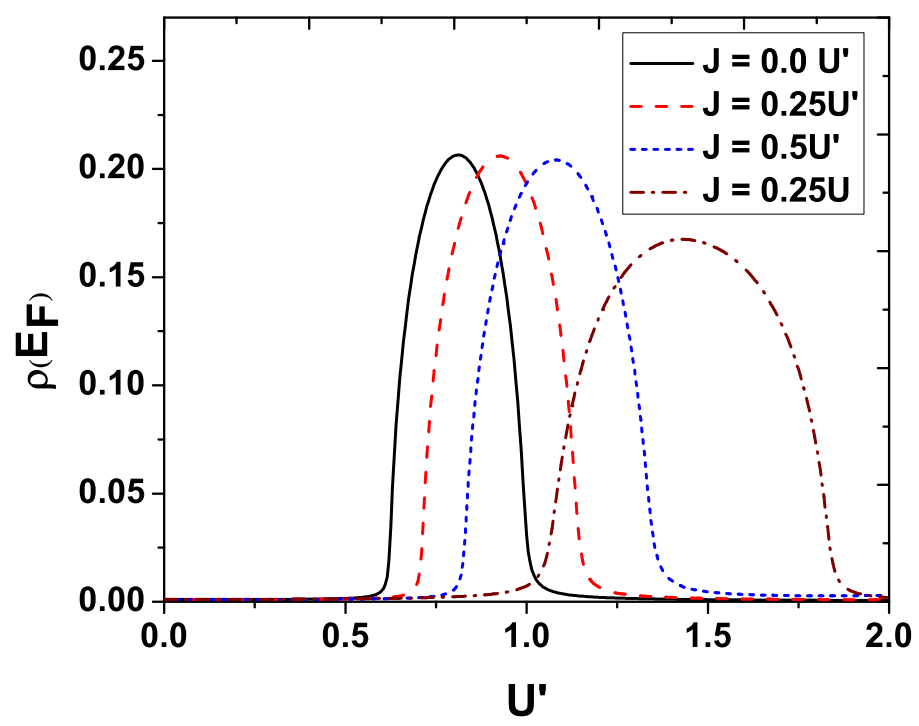

Fig. 4. DOS at the Fermi level as a function of $U^{\prime}$ for $U=1.5 W$ and different values of $J$.

only slightly changes the DOS near Fermi level, however, it does make different at the band edges. As a result, see Fig. 2, it inconsiderably changes the Mott transition which is characterized by the DOS at Fermi level. That means CPA result is in very good agreement with dynamical mean-field plus iterative perturbation theory by Kajueter and Kotliar [5]. By means of a generalized mean-field approximation, Didukh et al. [11] obtained similar results, except the fact that the effect of the band degeneracy was stronger than that predicted by DMFT and CPA.

When $U>1.0 \mathrm{~W}$ both the single-band and two-band models are in isulating phase. Fig. 3 shows how the groundstate of the two-band system changes when varying $U^{\prime}$ for $J=0$ and different values of $U$. For $U=0.5 W$ fixed, inscreasing $U^{\prime}$ from 0 , the system will undergo a metal-insulator transition once $U^{\prime}$ exceeds a critical value. On the other hand, for $U=1.5 \mathrm{~W}$, the system goes through a small metallic region in the insulating domain when inscreasing $U^{\prime}$, i.e., here are two distinct Mott-Hubbard transitions. The latter has been obtained within DMFT (exact diagonalization as the inpurity solvers) by Koga et al. [12,13] who argued that orbital fluctuations induced by the interband Coulomb interaction drives the system to the metallic phase. Fig. 4 shows effect of the Hund's exchange interaction $J$ on the Mott transitions of the system changes when varying $U^{\prime}$ for $U=1.5 \mathrm{~W}$. It is seen that the effect of the Hunds coupling is to broaden and shift the metallic peak to higher values of the interorbital interaction. 


\section{CONCLUSIONS}

We have applied coherent potential approximation for the half-filled isotropic degenerate two-band Hubbard model to study Mott-Hubbard transitions. We found that the orbital degeneracy slightly reduces the Mott-Hubbard critical value $U_{C}$. Furthermore, for $U$ large enough, the system goes through a small metallic region in the insulating domain when inscreasing $U^{\prime}$, i.e., here are two distinct Mott-Hubbard transitions. This result has also been obtained within DMFT by Koga et al. [12,13]. We showed that CPA results are in good agreement with DMFT. However, CPA requires less computer work, that enables one to consider all possible values of the system parameters. The results here can be extended to the anisotropic multiband model to study the so-called orbital-selective Mott transition, which has been received much of attention during the past few years. This is left to future work.

\section{ACKNOWLEDGMENT}

One of the authors (D. A. LE) would like to thank the HNUE project under the code SPHN-12-114 for financial support.

\section{REFERENCES}

[1] M. J. Rozenberg et al., Phys. Rev. Lett. 75 (1995)105.

[2] G. Moeller, Phys. Rev. B53 (1996) 16214.

[3] H. Hasegawa, J. Phys. Soc. Jpn. 66 (1997) 1391.

[4] Y. Ono, P. Potthoff, S. Bulla, Phys. Rev. B67 (2003) 035119.

[5] H. Kajueter, G. Kotliar, Int. J. Mod. Phys. B11 (1997) 729 .

[6] B. Velicky, S. Kirkpatrick and H. Ehrenreich, Phys. Rev. 175 (1968) 747.

[7] F. Ducastelle, J. Phys. C: Sol. Stat. Phys. 7 (1974) 1795.

[8] C. Lacroix-Lyon-Caen and M. Cyrot, Sol. Stat. Com. 21 (1977) 837.

[9] F. Gebhard, The Mott MetalInsulator Transition: Models and Methods (New York: Springer) 1997.

[10] J. van der Rest and F. Brouers, Phys. Rev. B24 (1981) 450.

[11] L. Didukh et al., Phys. Rev. B61 (2000) 7893.

[12] A. Koga et al., Phys. Rev. B66 (2002) 165107.

[13] A. Koga et al., J. Phys. Soc. Jpn. 72 (2003) 1306.

Received 31 October 2011. 